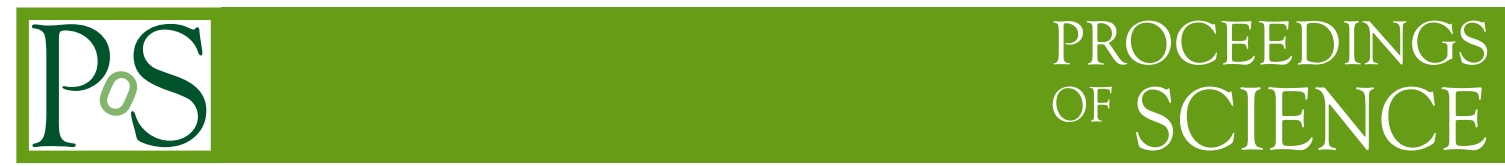

\title{
Schwarz maps for the hypergeometric function
}

\section{Masaaki Yoshida}

${ }^{a}$ Kyushu University,

Fukuoka 819-0395, Japan

E-mail: myoshida1948@jcom.home.ne.jp

In the former part of the talk, we start from a review of the classical theory of the Schwarz map for the Gauss hypergeometric function, and state a higher dimensional analogue. The intersection forms plays an essential role. In the latter part, we describe the hyperbolic Schwarz map for the Gauss hypergeometric function.

MathemAmplitudes 2019: Intersection Theory \& Feynman Integrals (MA2019)

18-20 December 2019

Padova, Italy 


\section{Introduction}

I am a mathematician. I have been interested in the hypergeometric function. Today I would like to speak about my love to this function, especially about the so-called Schwarz map.

Consider the hypergeometric differential equation

$$
x(1-x) u^{\prime \prime}+\{c-(a+b+1) x\} u^{\prime}-a b u=0, \quad E(a, b, c)
$$

where $\{a, b, c\}$ are complex parameters. This equation is defined on $\boldsymbol{C}-\{0,1, \infty\}$, and has mild singularities at $x=0,1$ and $\infty$. The hypergeometric series

$$
\sum_{n=0}^{\infty} \frac{(a)_{n}(b)_{n}}{(c)_{n} n !} x^{n}, \quad\left(a_{n}\right)=a(a+1) \cdots(a+n-1)
$$

is a typical solution. The Schwarz map of the hypergeometric differential equation is defined by

$$
s: X=C-\{0,1\} \ni x \longmapsto u_{0}(x): u_{1}(x) \in \boldsymbol{P}^{1},
$$

where $u_{0}$ and $u_{1}$ are linearly independent solutions of $E(a, b, c)$ and $\boldsymbol{P}^{1}$ is the complex projective line. The Schwarz map was studied by Schwarz in the end of 19th century when the parameters $(a, b, c)$ are real.

\section{Invariant forms and intersection matrices}

\subsection{Gauss hypergeometric equation}

The solutions of the equation are in general not single valued. If we analytically continue the solutions along a loop $\gamma$ starting and ending at, say $1 / 2$, then

$$
\left(u_{0}, u_{1}\right) \text { changes into }\left(u_{0}, u_{1}\right) M_{\gamma}
$$

where $M_{\gamma} \in \mathrm{GL}(2)$ is called a circuit matrix along $\gamma$. The circuit matrices form a group called the monodromy group of $E(a, b, c)$ with respect to the basis $\left\{u_{0}, u_{1}\right\}$. If we choose solutions represented by Euler integrals

$$
\begin{gathered}
u_{0}(x)=\int_{0}^{x} \omega, \quad u_{1}(x)=\int_{1}^{x} \omega, \\
\omega \quad=t^{b-c}(1-t)^{c-a-1}(x-t)^{-b} d t,
\end{gathered}
$$

it is easy to write down explicitly a set of generators of the monodromy group Monod. The entries of the generators are rational functions in $\left\{e^{2 \pi i a}, e^{2 \pi i b}, e^{2 \pi i c}\right\}$.

It is classically well-known that if $a, b, c$ are real, then there is a hermitian matrix $H$, unique up to constant multiple, such that

$$
M H M^{*}=H, \quad M \in \text { Monod. }
$$

Though the solutions $u_{0}$ and $u_{1}$ are not single valued, the quantity

$$
\left(u_{0}, u_{1}\right) H\left(\frac{\overline{u_{0}}}{\overline{u_{1}}}\right)
$$


is single valued; so it is often called the invariant hermitian form. This form determines the image of the Schwarz map.

Typical example: The Schwarz map $s$ of $E(1 / 2,1 / 2,1)$ sends $\boldsymbol{C}-\{0,1, \infty\}$ to a domain, projectively equivalent to a disc, which is determined by the invariant form. The inverse of the Schwarz map is the famous automorphic function $\lambda$. The image of $s$ looks like the wappen of the ICM.

Since a set of generators of Monod is easy to get, one can only to solve the above equation $M H M^{*}=H$ in $H$. The computation is not transparent nor enjoyable, but not so complicated and anyway one can solve to get $H$.

But why such $H$ exists?

For the hypergeometric equation $E(a, b, c)$ there is no need to ask so, because we have an explicit solution. For a given linear ordinary differential equation, and a basis $\left(u_{1}, u_{2}, \ldots\right)$ of solutions, is there a hermitian form $H$ such that

$$
\left(u_{1}, u_{2}, \ldots\right) H\left(u_{1}, u_{2}, \ldots\right)^{*}
$$

is single valued? Such $H$ does not exist for most equations.

\subsection{A high dimensional generalization of the hypergeometric equation}

About thirty years ago I encountered a linear integrable system of partial differential equations sometimes called the Aomoto-Gelfand hypergeometric system

$$
E(3,6 ; a), a=\left(a_{1}, \ldots, a_{6}\right), \quad a_{1}+\cdots+a_{6}=3 .
$$

It admits a hypergeometric type series solution, which I do not show here, and solutions represented by Euler integrals

$$
u(x)=\int_{D} \prod_{j=1}^{5} \ell_{j}(x)^{a_{j}-1} d s_{1} d s_{2}, \quad x=\left(x_{1}, \ldots, x_{4}\right), \quad \ell_{j}(x): \text { linear in }\left(s_{1}, s_{2}\right) .
$$

Here $(3,6)$ stands for (the dimension of $s$-space plus 1 , the number of liner forms $\ell_{j}$ plus 1 ). ${ }^{1}$ The system $E(3,6, a)$ is of rank 6 in 4 variables. This system is of rank 6 , because five lines in general position in the real plane cut out six bounded chambers. Note that three points on the real line cut out two bounded intervals.

The Schwarz map is defined by

$$
s: C^{4}-D \ni x \mapsto u_{0}(x): \cdots: u_{5}(x) \in \mathbb{P}^{5},
$$

${ }^{1}$ The Gauss hypergeometric equation is often written as

$$
E(2,4, a), a=\left(a_{1}, \ldots, a_{4}\right), \quad a_{1}+\cdots+a_{4}=2 ;
$$

This admits solutions represented by integrals

$$
\int s^{a_{1}-1}(s-1)^{a_{2}-1}(s-x)^{a_{3}-1} d s .
$$




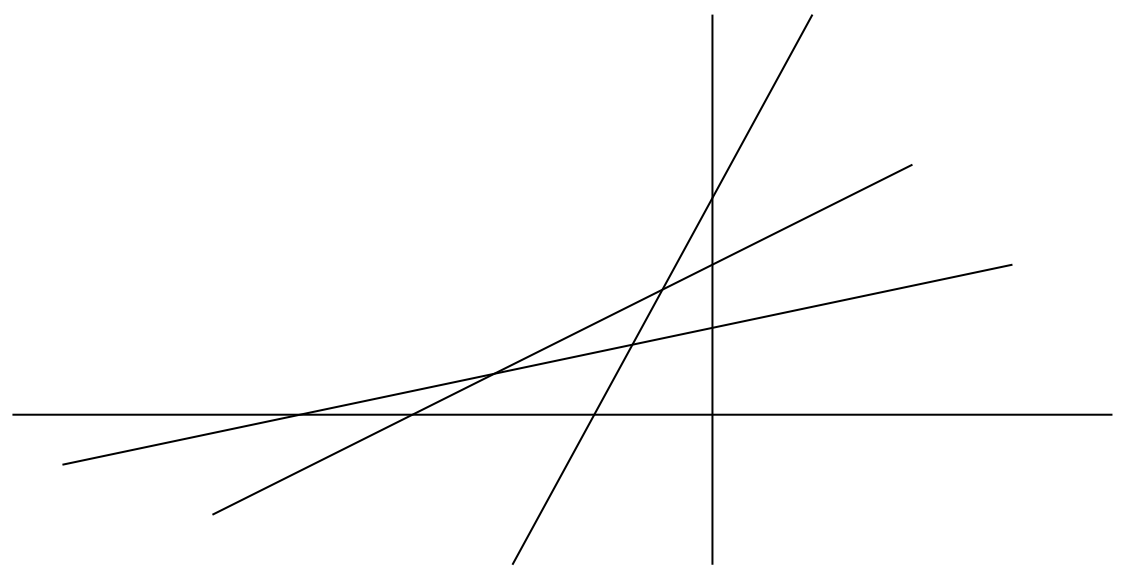

Figure 1: Five lines cut out six bounded chambers

where $D$ is a divisor, the singular locus of the system. I and my collaborators are interested in the special case $E(3,6 ; 1 / 2, \ldots, 1 / 2)$. In this case, there is an invariant hermitian form $H$, and the image of $s$ is determined as

$$
\left(z_{0}, \ldots, z_{5}\right) H^{t}\left(z_{0}, \ldots, z_{5}\right)=0, \quad\left(z_{0}, \ldots, z_{5}\right) H\left(z_{0}, \ldots, z_{5}\right)^{*}>0,
$$

the so-called type IV symmetric space. The inverse map is an automorphic map, which is not the subject today ([1]). Note that the typical example of Schwarz map in $\S 1.1$ is by $E(2,4 ; 1 / 2, \ldots, 1 / 2)$; so in a sense this is a natural generalization. By the way, $E(4,8 ; 1 / 2, \ldots, 1 / 2)$ does not work; life is not so easy.

We wondered whether there is an invariant hermitian form for general real parameters $a_{1}, \ldots, a_{6}$. We found a set of generators $M_{j}$ of the Monodromy group of $E(3,6 ; a)$; it is a hard work, made by my friends. And solved the system of linear equations

$$
M_{j} H M_{j}^{*}=H, \quad j=1,2, . .
$$

It is a system of linear equations, undergraduate math. But we have so many unknowns. Of course, we can ask a machine to do it. Younger generation do not know how primitive personal computer was 30 years ago. Machine was mainly used to check manual computation. One of my collaborators made the computations. Computation was long, but the solution existed, uniquely up to a multiplicative constant, and the result was surprisingly simple.

\subsection{Intersection forms}

So we thought there must be a reason for the invariant form to exist. By the way, for a basis of solutions, we can choose local solutions, that is, solutions expressed by hypergeometric series. But we can choose also solutions expressed by integrals. And we got an idea that if we can evaluate an intersection number $\bar{D}_{i} \cdot D_{j}$ for two paths/domains $D_{i}$ and $D_{j}$ of integration of two bases $u_{i}$ and $u_{j}$, the intersection matrix

$$
I:=\left(\bar{D}_{i} \cdot D_{j}\right)=\left(\begin{array}{c}
\bar{D}_{1} \\
\bar{D}_{2} \\
\vdots
\end{array}\right)\left(D_{1}, D_{2}, \ldots\right)
$$


is naturally invariant under small topological changes of the domains, so invariant under the monodromy group:

$$
M^{*} I M=I, \quad M \in \text { Monod }
$$

We found that

$$
H=I^{-1},
$$

so easy, so obvious. The assumption that the parameters $a_{j}$ are real is not necessary if we introduce the operator $a_{j} \rightarrow-a_{j}$; the dual of the local system, if you like. Anyway, when we found that this is really true we jumped up with joy ([2]). Long computation to get $H$ we do not need! Just evaluation of intersection numbers!

Today's audience know well how to evaluate these, I think, because on the poster of this conference, I found a fundamental example:

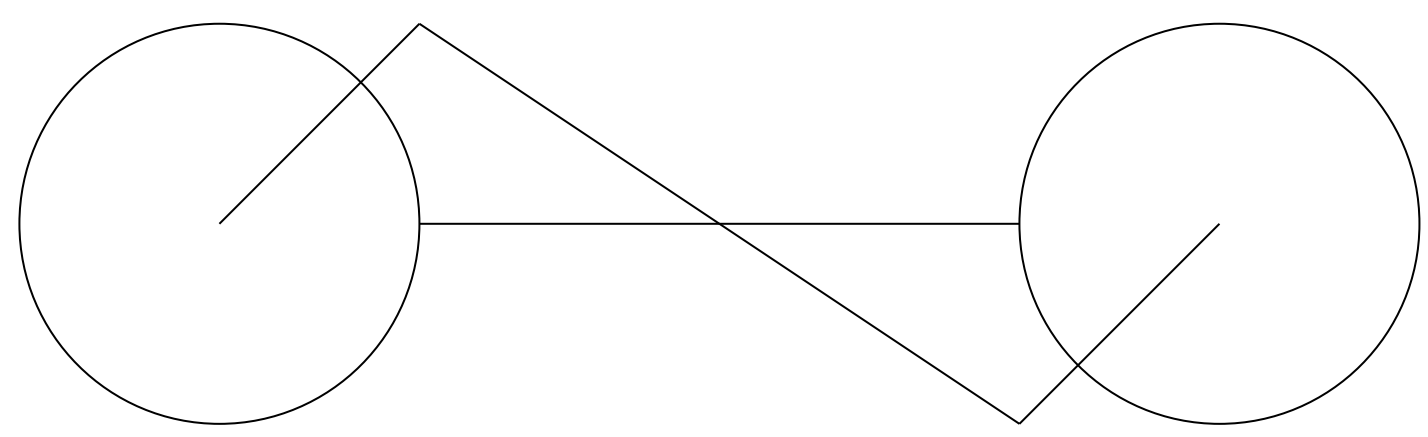

Figure 2: Evaluating an intersection number

I and my collaborators were happy to find a way to evaluate these intersection numbers. But among mathematicians this was and has been not popular at all. So when I got an email from Sebastian Mizera two years ago that this is useful for quantum field theory, I was just surprised, thank you.

\section{Hyperbolic Schwarz map for the Gauss hypergeometric equation}

After the work on the Schwarz map by himself, many kinds of high-dimensional versions are studied analytically, algebro-geometrically and arithmetically, in these decades. But I have had a slight reservation about the Schwarz map $s$ : Its target seems not to be exactly the correct one, because even if the monodromy group of $s$, the projective monodromy group of the equation, is discrete in $\mathrm{PGL}_{2}(\boldsymbol{C})$, for example $\mathrm{PGL}_{2}(\boldsymbol{Z}[i])$, it does not, in general, act properly discontinuously on any non-empty open set of the target $\boldsymbol{P}^{1}$, and so the image would be chaotic.

The 1-dim complex projective space $\boldsymbol{P}^{1}$ is also called the Riemann sphere; yes, it is a sphere. The group $\mathrm{PGL}_{2}(\boldsymbol{C})$ acts naturally on the inside of the sphere not the skin! Inside of the sphere, the ball, is the 3-dim hyperbolic space $\boldsymbol{H}^{3}$ equipped with the motion group $\mathrm{PGL}_{2}(\boldsymbol{C})$.

For years, I dreamed a correct Schwarz map with target $\boldsymbol{H}^{3}$, which should be called the hyperbolic Schwarz map. 


\subsection{Hyperbolic Schwarz map}

About ten years ago, I got it ([4]), once it is found, it is simple and natural, of course. It is defined as follows: Change the equation $E(a, b, c)$ into the so-called $S L$-form:

$$
u^{\prime \prime}-q(x) u=0,
$$

and transform it to the matrix equation

$$
\frac{d}{d x}\left(\begin{array}{c}
u \\
u^{\prime}
\end{array}\right)=\Omega\left(\begin{array}{c}
u \\
u^{\prime}
\end{array}\right), \quad \Omega=\left(\begin{array}{cc}
0 & 1 \\
q(x) & 0
\end{array}\right) .
$$

We now define the hyperbolic Schwarz map, denoted by $\mathcal{S}$, as the the map

$$
\mathcal{S}: X \ni x \longmapsto U(x)^{*} U(x) \in \operatorname{Her}^{+}(2) / \boldsymbol{R}^{+}=: \boldsymbol{H}^{3},
$$

where

$$
U(x)=\left(\begin{array}{ll}
u_{0} & u_{1} \\
u_{0}^{\prime} & u_{1}^{\prime}
\end{array}\right)(x)
$$

is a fundamental solution of the system, $\operatorname{Her}^{+}(2)$ the space of positive-definite Hermitian matrices of size 2 , and $\boldsymbol{R}^{+}$the multiplicative group of positive real numbers.

Note that the target of the hyperbolic Schwarz map is the hyperbolic 3-space $\boldsymbol{H}^{3}$, whose boundary is $\boldsymbol{P}^{1}$, which is the target of the Schwarz map. In this sense, our hyperbolic Schwarz map is a lift-to-the-air of the Schwarz map. Note also that the monodromy group of the system acts naturally on $\boldsymbol{H}^{3}$ :

$$
W=U(x)^{*} U(x) \rightarrow M^{*} W M, \quad M \in \text { Monod. }
$$

The image surface (of $X$ under $\mathcal{S}$ ) has the following geometrically nice property:

- It has singularities along the image of the curve

$$
\{x \in C ;|q|=1\} .
$$

Generic singularities of the image surface are cuspidal edges and swallowtail singularities. Singularities are welcome, because singular points are points carrying more information than ordinary points.

- The classical Schwarz map $s$ is recovered as follows: from an image point $S(x)$, extend the normal as a geodesic curve to hit the ideal boundary $\mathbb{P}^{1}$ at two points: one is the Schwarz image $s(x)$, and the other is the derived Schwarz image, where derived Schwarz map is defined by

$$
d s: X \ni x \longmapsto u_{0}^{\prime}(x): u_{1}^{\prime}(x) \in \mathbb{P}^{1} .
$$

- If the projective monodromy group is discrete in $\operatorname{PGL}(2, \boldsymbol{C})$, then the image is a closed surface in $\boldsymbol{H}^{3}$. 


\subsection{Examples}

To conclude this talk, I show the images under the hyperbolic Schwarz map $\mathcal{S}$ of the hypergeometric equation when the monodromy group is a dihedral group and a Fuchsian group. As a dessert, I also show it for the Airy equation, which is the most confluent hypergeometric equation. You can find many swallowtails; they look so nice.

You see, I am doing mathematics just for fun, thank you.
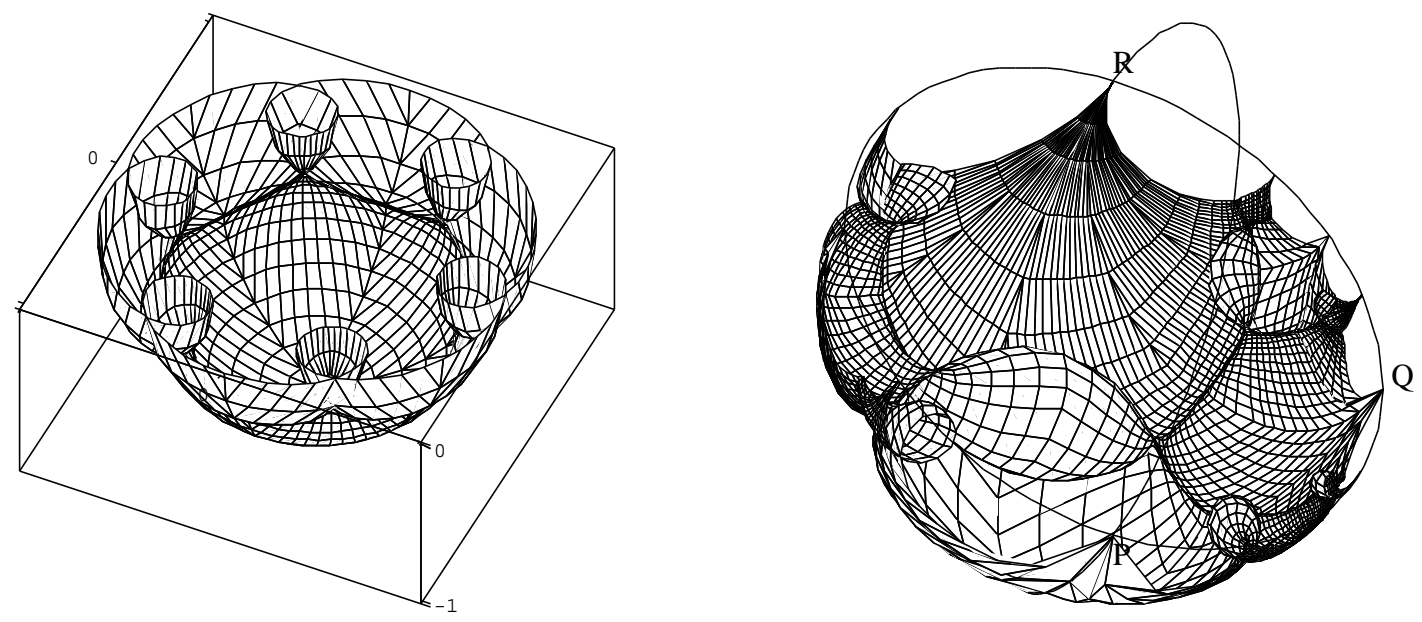

Figure 3: Image under $\mathcal{S}$ : Hypergeometric equations with a dihedral and a Fuchsian monodromy groups

\section{References}

[1] K. Matsumoto, T.Sasaki and M. Yoshida, The monodromy of the period map of a 4-parameter family of K3 surfaces and the hypergeometric function of type (3,6), Internat J Math 3(1992).

[2] M. Kita and M. Yoshida, Intersection theory for twisted cycles I, II, Math. Nachr. 166, 168(1994).

[3] K. Mimachi and M. Yoshida, Intersection numbers of twisted cycles and the correlation functions of the conformal field theory, Commun. Math. Phys. 243(2003).

[4] T. Sasaki, K. Yamada and M. Yoshida, Hyperbolic Schwarz map for the hypergeometric function, Experiment. Math. 17(2008).

[5] S. Fujimori, M. Noro, K. Saji, T. Sasaki and M. Yoshida, Schwarz maps for the hypergeometric differential equations, Internat J Math. 26(2015). 


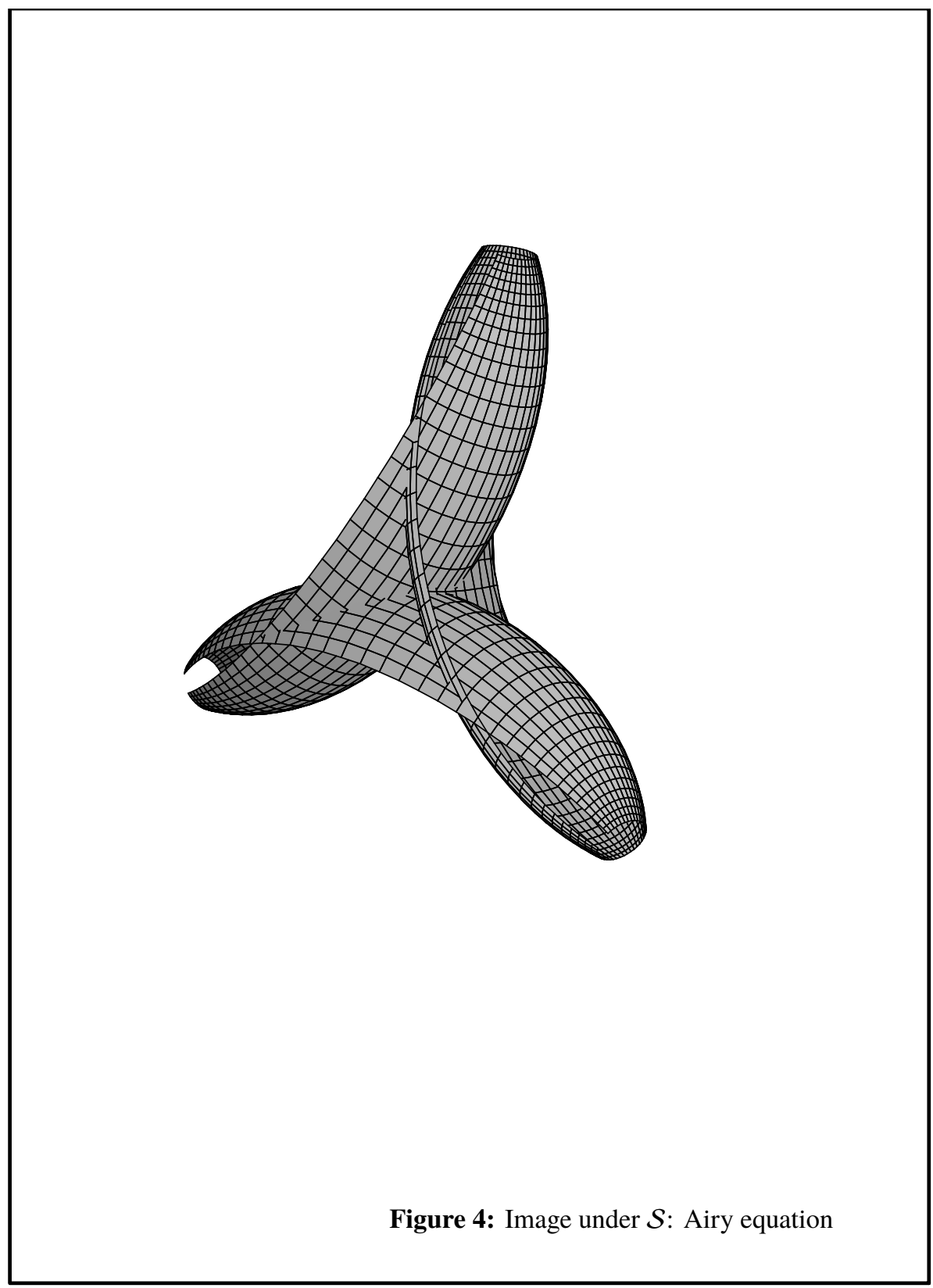

